\title{
Structure-activity Relationship Analysis for Antimicrobial Activities of Tryptanthrin Derivatives Using Quantum Chemical Calculations
}

\author{
Jun KAWAKAMI ${ }^{\mathrm{a},}{ }^{,}$, Hiroko KAKINAMI ${ }^{\mathrm{a}}$, Noriyuki MATSUSHIMAª ${ }^{\mathrm{a}}$ Akio NAKANE $^{\mathrm{b}}$, \\ Haruo KITAHARA ${ }^{c}$, Masahiko NAGAKI ${ }^{a}$, Shunji ITO ${ }^{a}$
}
${ }^{a}$ Graduate School of Science and Technology, Hirosaki University, 3 Bunkyo-cho, Hirosaki, Aomori 036-8561, Japan
${ }^{\mathrm{b}}$ Graduate School of Medicine, Hirosaki University, 5 Zaifu-cho, Hirosaki, Aomori 036-8562, Japan
${ }^{c}$ Department of Natural Science, Faculty of Education, Hirosaki University,
1 Bunkyo-cho, Hirosaki, Aomori 036-8560, Japan
*e-mail: jun@cc.hirosaki-u.ac.jp

(Received: September 10, 2012; Accepted for publication: February 8, 2013; Advance publication: April 6, 2013)

Tryptanthrin (T) and eight of its monosubstituted derivatives (T2NH $2, \mathbf{T 2 C l}, \mathbf{T 2 B r}, \mathrm{T}_{2} \mathrm{NO}_{2}, \mathbf{T 8 O M e}, \mathbf{T 8 M e}, \mathbf{T 8 F}$, and $\mathbf{T 8 B r}$ ) were synthesized, and their antimicrobial activities were investigated against a fungus (Malassezia furfur) and a gram-positive bacterium (methicillin-resistant staphylococcus aureus, MRSA). Antimicrobial activities of these derivatives were influenced by the substituents on tryptanthrin, with the halogen-substituted tryptanthrin derivatives (T2Cl, T2Br, T8F, and T8Br) showing the highest potency against $M$. furfur and MRSA. Therefore, semiempirical molecular orbital calculations (PM3) were performed on $\mathbf{T}$ and its eight derivatives to investigate the cause of the differences in their antimicrobial activities. The results of the calculations showed that antimicrobial activities could be related to the electrophilicity of the carbonyl carbon of the five-membered ring.

Keywords: PM3, Antimicrobial activity, Tryptanthrin, MRSA, M. furfur

\section{Introduction}

Tryptanthrin (T, Figure 1) is a weakly basic alkaloid found in a number of plant species [1]. This compound exhibits antimicrobial activity against various pathogenic bacteria and fungi [2]. In particular, the antifungal activity of tryptanthrin against Malassezia furfur, which is the causative fungus of atopic dermatitis, is significant [3]. Tryptanthrin is also effective in the treatment of contact dermatitis (delayed-type allergy) [4]. Therefore, its use as a therapeutic drug for conditions such as

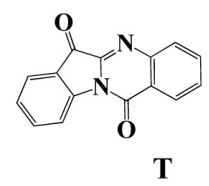

atopic dermatitis or in cosmetics is anticipated. However, few reports describe the properties of chemosynthetic tryptanthrin derivatives, which are not found in nature. In particular, there are few studies on the antifungal activities of tryptanthrin derivatives against $M$. furfur [3]. Therefore, we synthesized eight different tryptanthrin derivatives (Figure 2) and investigated their antimicrobial properties against a fungus (M. furfur) and a gram-positive bacterium (methicillin-resistant Staphylococcus aureus, MRSA).

The antifungal and antibacterial activities of the derivatives were influenced by tryptanthrin substituents, with halogen-substituted tryptanthrin derivatives (T2Cl, T2Br, T8F, and T8Br) showing the highest potency against M. furfur and MRSA [5]. Subsequently, semiempirical molecular orbital calculations were performed on tryptanthrin derivatives to investigate the structure-activity relationships of the antimicrobial activities.

Figure 1. Structure of tryptanthrin (T). 

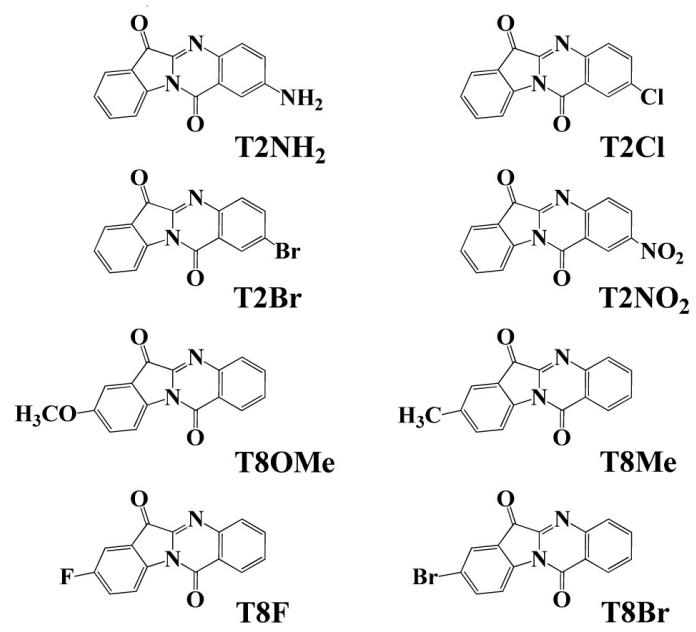

Figure 2. Structures of the eight tryptanthrin derivatives.

\section{Experiment}

\subsection{Synthesis}

Material data and preparation of tryptanthrin (T) and eight of its derivatives $\left(\mathbf{T}_{2} \mathrm{NH}_{2}, \mathbf{T 2 C l}, \mathbf{T} 2 \mathrm{Br}, \mathbf{T}_{2} \mathrm{NO}_{2}, \mathbf{T} 8 \mathrm{OMe}, \mathbf{T} 8 \mathrm{Me}\right.$, $\mathbf{T 8 F}$, and $\mathbf{T 8 B r}$ ) have been reported [5].

\subsection{Antibacterial and antifungal activity test}

The antifungal and antibacterial activities of the derivatives against a fungus (M. furfur) and a gram-positive bacterium (MRSA) were investigated in vitro, using the agar plate dilution method described by the Japanese Society of Chemotherapy [6-9]. A bacterial culture (developed over 2-3 days for M. furfur or overnight for MRSA) was diluted with malt extract broth ( $36 \mathrm{~g} / \mathrm{L}$ in distilled water; Wako Pure Chemical Industries, Ltd.) containing Tween-40 $(10 \mathrm{~g} / \mathrm{L}$ in distilled water; Wako Pure Chemical Industries, Ltd.), monoolein ( $2 \mathrm{~g} / \mathrm{L}$ in distilled water; Tokyo Chemical Industry Co., Ltd.), oxgall $(20 \mathrm{~g} / \mathrm{L}$ in distilled water; Wako Pure Chemical Industries, Ltd.), peptone $(6 \mathrm{~g} / \mathrm{L}$ in distilled water; Wako Pure Chemical Industries, Ltd.), and glycerol (2 g/L in distilled water; Wako Pure Chemical Industries, Ltd.) for M. furfur and with Mueller-Hinton broth $(21 \mathrm{~g} / \mathrm{L}$ in distilled water; Becton, Dickinson and Co.) for MRSA to a density of $1.0 \times 10^{6}$ colony-forming units $(\mathrm{CFU}) / \mathrm{mL}$. The compounds for testing $\left(\mathbf{T}, \mathbf{T} 2 \mathrm{NH}_{2}, \mathbf{T} 2 \mathrm{Cl}, \mathbf{T} 2 \mathrm{Br}, \mathbf{T} 2 \mathrm{NO}_{2}, \mathbf{T} 8 \mathrm{OMe}\right.$, T8Me, T8F, and T8Br) were dissolved in DMSO (Wako Pure Chemical Industries, Ltd.) and diluted with malt extract broth containing Tween-40, monoolein, oxgall, peptone, and glycerol to a concentration of 4-160 $\mu \mathrm{g} / \mathrm{mL}$ for $M$. furfur and with Mueller-Hinton broth to a concentration of $0.1-100 \mu \mathrm{g} / \mathrm{mL}$ for MRSA. Thereafter, each Petri dish was inoculated with the fun-
Table 1. Antimicrobial activity (MIC) of tryptanthrin (T) and its eight derivatives $\left(\mathbf{T} 2 \mathrm{NH}_{2}, \mathbf{T} 2 \mathrm{Cl}, \mathbf{T} 2 \mathrm{Br}, \mathbf{T}_{2} \mathrm{NO}_{2}, \mathbf{T 8 O M e}\right.$, T8Me, T8F, and T8Br) against $M$. furfur and MRSA.

\begin{tabular}{|c|c|c|}
\hline & \multicolumn{2}{|c|}{$\mathrm{MIC} / \mu \mathrm{g} \mathrm{mL} \mathrm{m}^{-1}$} \\
\hline & M. furfur & MRSA \\
\hline $\mathbf{T}$ & 4 & 0.5 \\
\hline $\mathrm{T} 2 \mathrm{NH}_{2}$ & 20 & 2.0 \\
\hline $\mathrm{T} 2 \mathrm{Cl}$ & 4 & 0.1 \\
\hline T2Br & 4 & 0.3 \\
\hline $\mathrm{T}_{2} \mathrm{NO}_{2}$ & $>160$ & $>100$ \\
\hline T8OMe & $>120$ & 2.5 \\
\hline T8Me & $>120$ & 0.5 \\
\hline T8F & 1 & 0.1 \\
\hline T8Br & 2.5 & 0.1 \\
\hline
\end{tabular}

$\mathrm{MIC}=$ minimum inhibitory concentration in $\mu \mathrm{g} / \mathrm{mL}$.

gal or bacterial suspension and incubated at $37^{\circ} \mathrm{C}$ for $2-4$ days for $M$. furfur and $24 \mathrm{~h}$ for MRSA. The lowest concentration at which there was no visible growth was considered as the minimum inhibitory concentration (MIC).

\subsection{Calculation procedure}

The molecular modeling study was performed using the SPARTAN '10 software package (Wavefunction Inc., Irvine, CA, 2000). The minimum energy conformation of the tryptanthrin derivatives was obtained by the MMFF of the molecular mechanics calculation. We evaluated the electronic properties of the MMFF minimum energy conformation by using semiempirical molecular orbital calculations (PM3). To study the structure-activity relationships, we evaluated electronic properties of the lowest unoccupied molecular orbital (LUMO), the LUMO map, and the electrostatic potential map.

\section{Results and discussion}

\subsection{Antifungal and antibacterial activities of Tryptanthrin and its eight deriva- tives}

The antibacterial activities of tryptanthrin $(\mathrm{T})$ and its derivatives $\left(\mathbf{T}_{2} \mathrm{NH}_{2}, \mathbf{T} 2 \mathrm{Cl}, \mathbf{T 2 B r}, \mathbf{T}_{2} \mathrm{NO}_{2}\right.$, T8OMe, T8Me, T8F, and T8Br) were investigated on a fungus ( $M$. furfur) and a grampositive bacterium (MRSA) in culture. MICs for M. furfur and MRSA are shown in Table 1.

The antifungal and antibacterial activities are enhanced for small MIC values. The MIC at which a molecule exerted antimicrobial activity was defined as less than $800 \mu \mathrm{g} / \mathrm{mL}$ in accordance with the criterion specified by the Japanese Society of Chemotherapy [7-9]. Most tryptanthrin derivatives showed high antifungal (M. furfur) and antibacterial activities (MRSA). The antibacterial activity for MRSA was higher than the antifungal activity for M. furfur. The higher potency of the derivatives 
against the gram-positive bacterium (MRSA) compared with their potency against the fungus (M. furfur) can be attributed to the structural differences between the two species. The MIC order for $M$. furfur was $\mathbf{T 8 F}<\mathbf{T 8 B r}<\mathbf{T} 2 \mathbf{C l}=\mathbf{T} 2 \mathbf{B r}=\mathbf{T}<\mathbf{T} 2 \mathbf{N H}_{\mathbf{2}}<<$ T8OMe, T8Me, $\mathbf{T 2 N O}_{2}$, while for MRSA it was $\mathbf{T 8 F}=\mathbf{T 8 B r}=$ $\mathbf{T} 2 \mathrm{Cl}<\mathbf{T} 2 \mathrm{Br}<\mathbf{T}=\mathbf{T} 8 \mathrm{Me}<\mathbf{T} 2 \mathbf{N H}_{2}<\mathbf{T 8 O M e}<<\mathbf{T} 2 \mathbf{N O}_{2}$. For both M. furfur and MRSA, the antifungal and antibacterial activities of the halogen-substituted tryptanthrin derivatives were higher when compared to the other tryptanthrin derivatives. Considering the overall efficacy, $\mathbf{T 8 F}$ was the most potent of all the tested compounds with an MIC of $1 \mathrm{mg} / \mathrm{mL}$ for $M$. furfur and $0.1 \mathrm{mg} / \mathrm{mL}$ for MRSA. In contrast, the antibacterial and antifungal activities of $\mathbf{T} 2 \mathrm{NO}_{2}$, which has a nitro group at position 2 of tryptanthrin, were the lowest. The MIC of $\mathbf{T} 2 \mathbf{N O}_{2}$ was $>160 \mu \mathrm{g} / \mathrm{mL}$ for $M$. furfur and $>100 \mu \mathrm{g} / \mathrm{mL}$ for MRSA. Miconazole nitrate is currently used as a therapeutic drug for treating atopic dermatitis. Its MIC for $M$. furfur, which is the causative fungus of atopic dermatitis, is ca. $25 \mu \mathrm{g} / \mathrm{mL}$. The MICs of $\mathbf{T}$ and its halogen-substituted tryptanthrin derivatives $\mathbf{T 8 F}$, $\mathbf{T 8 B r}$, T2Cl, T2Br, and T for $M$. furfur ranged from 1 to $4 \mu \mathrm{g} / \mathrm{mL}$, and their antifungal activities were more than six times that of miconazole nitrate. T8F was found to be particularly effective.

\subsection{Structure-activity relationships of tryptanthrin and its eight derivatives}

Molecular level information obtained from semiempirical (PM3) quantum chemical calculations on the optimized electronic structure of compounds was used to explore the relationship between chemical structure and biological activity. Structure-activity relationships are based on the fundamental hypothesis that biological properties are functions of the molecular structure. It is reasonable to assume that certain electrophilic or nucleophilic sites of a molecule can only bind to nucleophilic or electrophilic sites of the receptor.

Previously, it was reported that the correlation between antileishmanial activity of tryptanthrin derivatives and the lowest unoccupied molecular orbital (LUMO) energy is significant, and the electron transfer ability from a receptor to the carbonyl carbon of a five-membered ring in the tryptanthrin moiety is presumably the crucial step in the mechanism of action of the compounds for antileishmanial activity [10]. The presence of a five-membered carbonyl moiety in the molecule appears to be the structural requirement for potent activity. Therefore, we investigated the correlation between the activity and LUMO energy. The plot of the LUMO energy versus MIC values is shown in Figures. 3 and 4; however, $\mathbf{T 2 N O}_{2}$, T8OMe, and T8Me against $M$. furfur and $\mathbf{T} 2 \mathbf{N O}_{2}$ against MRSA were removed from the plot because we cannot use them to establish the corresponding MICs. Figures 3 and 4 indicate that a more negative LUMO energy favors potent activity in the tryptanthrins. Although the plot of LUMO energy versus MIC values does not

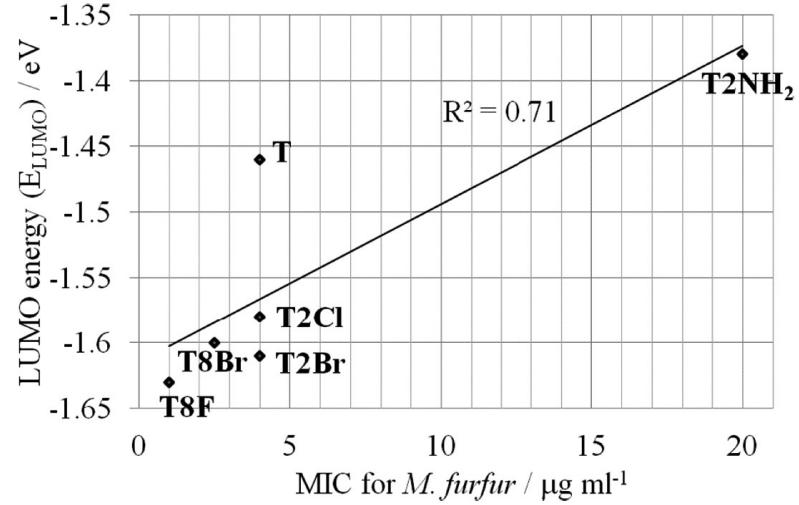

Figure 3. The lowest unoccupied molecular orbital (LUMO) energy against the antifungal activity of tryptanthrin $\left(r^{2}=0.71\right)$.

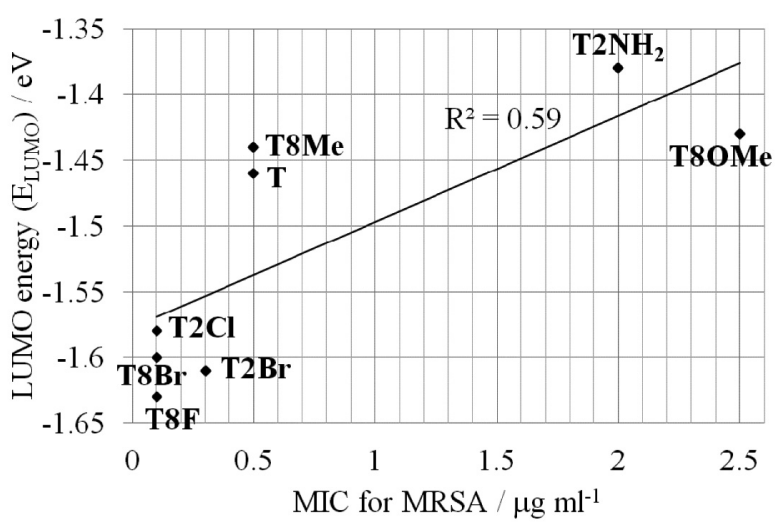

Figure 4. The lowest unoccupied molecular orbital (LUMO) energy against the antibacterial activity of tryptanthrin $\left(r^{2}=0.59\right)$

indicate an excellent linear correlation $\left(\mathrm{r}^{2}=0.71\right.$ for $M$. furfur; $\mathrm{r}^{2}=0.59$ for MRSA), the trend is apparent. Thus, the electron affinity of the molecule could be an important factor associated with potent activity. The molecular properties of $\mathbf{T}, \mathbf{T 8 F}$ and $\mathbf{T} 2 \mathrm{NO}_{2}$ retrieved by using semiempirical molecular orbital calculations (PM3) are shown in Figure 5 as typical examples. T8F was the most potent of all the tested compounds with an MIC of $1 \mu \mathrm{g} / \mathrm{mL}$ for M. furfur and $0.1 \mu \mathrm{g} / \mathrm{mL}$ for MRSA. T also showed antimicrobial activities with an MIC of $4 \mu \mathrm{g} / \mathrm{mL}$ for $M$. furfur and $0.5 \mu \mathrm{g} / \mathrm{mL}$ for MRSA, while $\mathbf{T} 2 \mathrm{NO}_{2}$ did not show antimicrobial activities against both M. furfur and MRSA. As shown in Figure 5b, the shape of the LUMO of the carbonyl carbon atom of the five-membered ring of $\mathbf{T 8 F}$ and $\mathbf{T}$ with high antimicrobial activities is large. In contrast, that of $\mathbf{T} \mathbf{2} \mathbf{N O}_{2}$ with no antimicrobial activity is smaller, and the shape of the next carbon atom of the carbonyl carbon atom of the five-membered ring is larger than that of the carbonyl carbon atom of the fivemembered ring. In the LUMO map, the electron-poor sites are indicated in blue, while the electron-rich sites are indicated 


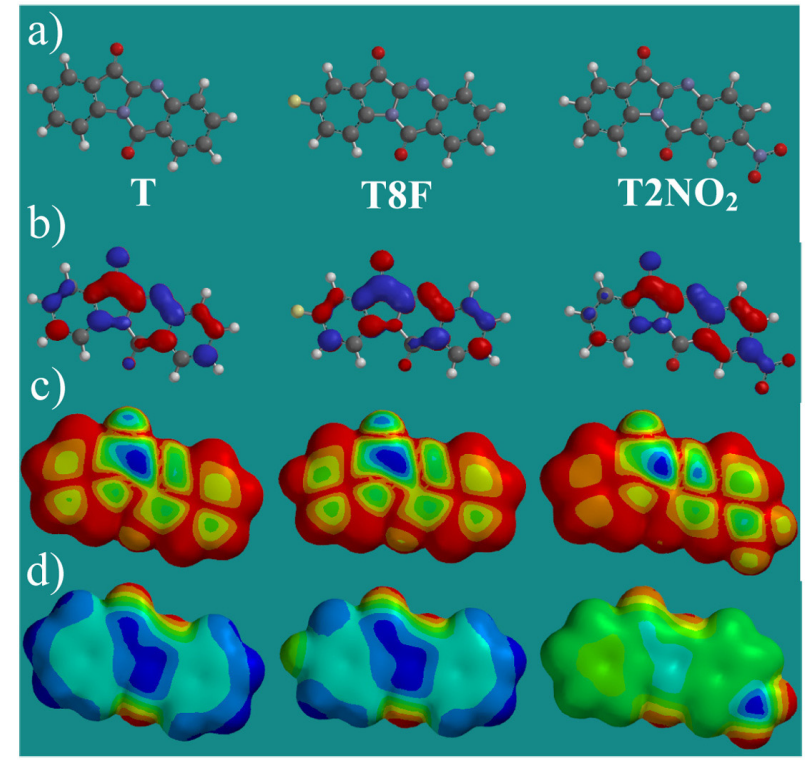

Figure 5. Molecular properties of T, T8F and $\mathbf{T} 2 \mathrm{NO}_{2}$ by semiempirical molecular orbital calculations (PM3); a) Molecular structures, b) molecular orbital of LUMO, c) LUMO maps and d) electrostatic potential maps.

in red. As shown in Figure 5c, the LUMO map of T8F and T shows intensely blue atoms over the carbonyl carbon atom, while that of $\mathbf{T} 2 \mathrm{NO}_{2}$ shows green atoms. The next carbon atom of the carbonyl carbon atom of the five-membered ring is intensely blue. The LUMO map shows one prominent site by the carbonyl group of the five-membered ring in tryptanthrin derivatives, the shape of the LUMO is found to be large and extended by the carbonyl carbon atom of the five-membered ring in the more potent analogs. The electrostatic potential map is an alternative approach for analyzing the electrostatic contribution to the binding of the receptor and drugs [11]. As shown in Figure 5d, the electrostatic potential map analysis revealed a lower electron density (blue color) over the carbonyl carbon atom of the five-membered ring of $\mathbf{T 8 F}$ and $\mathbf{T}$ than that observed in $\mathbf{T} 2 \mathbf{N O}_{2}$. Electron transfer from the receptor site to this carbonyl carbon seems a plausible path for the mechanism action of the compounds. According to the results of the theoretical calculations, the antifungal and antibacterial activities could be related to the electrophilicity of the carbonyl carbon of the five-membered ring.

\section{Conclusion}

Tryptanthrin (T) and its eight derivatives $\left(\mathbf{T} 2 \mathbf{N H}_{\mathbf{2}}, \mathbf{T} \mathbf{2} \mathbf{C l}\right.$, T2Br, $\mathrm{T2NO}_{2}$, T8OMe, T8Me, T8F, and T8Br) were synthesized, and their antibacterial properties and antifungal properties against a fungus ( $M$. furfur) and a gram-positive bacterium (MRSA) were investigated. The antifungal and antibacterial activities of the halogen-substituted tryptanthrin derivatives against $M$. furfur and MRSA were exceptional. Especially, T8F was the most potent of all the tested compounds with an MIC of $1 \mu \mathrm{g} / \mathrm{mL}$ for $M$. furfur and $0.1 \mu \mathrm{g} / \mathrm{mL}$ for MRSA. The semiempirical molecular orbital calculations (PM3) were performed on $\mathbf{T}$ and eight of its derivatives to investigate the cause of the differences in their antimicrobial activities. The results of the calculations showed that the antimicrobial activities could be related to the electrophilicity of the carbonyl carbon of the five-membered ring. The carbonyl groups of the five-membered rings in the tryptanthrin moiety and the electron transfer ability from a receptor could be a crucial step in the mechanism of action of these compounds.

This study was partly supported by a Grant for Priority Research Designated from the dean of the Faculty of Science and Technology of Hirosaki University (2008-2009) and partly by a Grant-in-Aid for Scientific Research (C) No. 22550068 from JSPS.

\section{References}

[1] A. Witt, J. Bergman, Curr. Org. Chem., 7, 659 (2003). [CrossRef]

[2] L. A. Mitscher, W. C. Wong, T. De Meulenere, J. Sulko, S. Drake, Heterocycles, 15, 1017 (1981). [CrossRef]

[3] H. Kitahara et al., Japan Patent Pending, 2003, Tokugan 2003-393219, Tokukai 2004-189732.

[4] H. Kitahara et al., Japan Patent Pending, 2012, 5023317.

[5] J. Kawakami, N. Matsushima, Y. Ogawa, H. Kakinami, A. Nakane, H. Kitahara, M. Nagaki, S. Ito, Trans. Mater. Res. Soc. Jpn., 36, 603 (2011).

[6] M. Nagaki, T. Narita, H. Ichikawa, J. Kawakami, A. Nakane, Trans. Mater. Res. Soc. Jpn., 36, 55 (2011).

[7] A. Nagayama, K. Yamaguchi, K. Watanabe, M. Tanaka, I. Kobayashi, Z. Nagasawa, J. Infect. Chemother., 14, 383 (2008). [Medline] [CrossRef]

[8] S. Arakawa, T. Matsui, S. Kamidono, Y. Kawada, H. Kumon, K. Hirai, T. Hirose, T. Matsumoto, K. Yamaguchi, T. Yoshida, K. Watanabe, K. Ueno, A. Saito, T. Teranishi, J. Infect. Chemother, 4, 97 (1998). [CrossRef]

[9] A. Saito, J. Infect. Chemother., 1, 83 (1995). [CrossRef]

[10] A. K. Bhattacharjee, D. J. Skanchy, B. Jennings, T. H. Hudson, J. J. Brendle, K. A. Werbovetz, Bioorg. Med. Chem., 10, 1979 (2002). [Medline] [CrossRef]

[11] A. M. R. Bernardino, H. C. Castro, I. C. P. P. Frugulhetti, N. I. V. Loureiro, A. R. Azevedo, L. C. S. Pinheiro, T. M. L. Souza, V. Giongo, F. Passamani, U. O. Magalhães, M. G. Albuquerque, L. M. Cabral, C. R. Rodrigues, Bioorg. Med. Chem., 16, 313 (2008). [Medline] [CrossRef] 